\title{
Upregulation of microRNA-155 promotes the migration and invasion of colorectal cancer cells through the regulation of claudin-1 expression
}

\author{
GUANG-JUN ZHANG* ${ }^{*}$, HUA-XU XIAO*, HONG-PENG TIAN, \\ ZUO-LIANG LIU, SHU-SEN XIA and TONG ZHOU
}

First Department of General Surgery, Institute of Hepatobiliary, Pancreatic and Intestinal Disease, The Affiliated Hospital of North Sichuan Medical College, Nanchong, Sichuan 637000, P.R. China

Received February 11,2013; Accepted April 8, 2013

DOI: $10.3892 /$ ijmm.2013.1348

\begin{abstract}
Human microRNA-155 (miR-155) has been demonstrated to regulate a variety of cellular functions, including epithelial-to-mesenchymal transition (EMT) by targeting multiple messenger RNAs (mRNAs). However, its role in colorectal cancer (CRC) remains unelucidated. Therefore, the aim of the present study was to investigate the effects of miR-155 on CRC cells. The expression level of miR-155 was quantified by quantitative real-time reverse transcriptase-PCR (qRT-PCR) in primary CRC tissues and normal adjacent mucosa. MTT, migration and invasion assays were used to examine the proliferation, migration and invasion of SW480 cells transfected with miR-155. The expression of miR-155 was significantly upregulated in the CRC tissues and the high expression of miR-155 correlated with an advanced clinical stage, lymph node and distant metastases. The ectopic expression of miR-155 enhanced the migration and invasive ability of the SW480 cells and altered their morphological appearance; however, cell proliferation was not affected. E-cadherin expression levels decreased, while ZEB1 expression levels increased in the SW480 cells overexpressing miR-155. Furthermore, the overexpression of miR-155 upregulated claudin-1 expression. Thus, our data suggest that miR-155 plays an important role in promoting CRC cell migration and invasion, at least in part through the regulation of claudin-1 expression and controlling metastasis in CRC.
\end{abstract}

Correspondence to: Dr Tong Zhou, First Department of General Surgery, Institute of Hepatobiliary, Pancreatic and Intestinal Disease, The Affiliated Hospital of North Sichuan Medical College, 63 Wenhua Road, Nanchong, Sichuan 637000, P.R. China

E-mail: zhoutong0088@163.com

*Contributed equally

Key words: miR-155, colorectal cancer, claudin-1, migration, invasion

\section{Introduction}

Colorectal carcinoma (CRC) is one of the leading causes of cancer-related mortality worldwide. The incidence and mortality rate of CRC in China have increased rapidly over the past several decades (1). A significant number of patients with CRC who undergo curative surgery develop local recurrence or distant metastasis, leading to shorter survival (2). A better understanding of the molecular mechanisms underlying metastasis is essential to facilitate the prevention and treatment of advanced CRC.

One of the key molecular steps in the process of distant metastasis includes epithelial-to-mesenchymal transition (EMT), which permits invasion and migration in various types of cancer $(3,4)$, and is associated with a poor prognosis in CRC (5). During EMT, epithelial cell-cell adhesion is decreased through the downregulation of adhesion molecules, such as E-cadherin, and the cells then acquire a spindleshaped, highly motile fibroblast-like phenotype $(6,7)$. Several mechanisms for E-cadherin transcriptional silencing during EMT have been suggested, including the direct inhibition by transcriptional repressors, such as Snail, Twist and zinc finger E-box-binding homeobox (ZEB)1 and ZEB2 (8-10).

MicroRNAs (miRNAs or miRs) are a large family of highly conserved short (approximately 22 nucleotides in length) singlestranded non-coding RNAs which regulate the translational inhibition of target messenger RNAs (mRNAs) by binding to their 3'-untranslated region (UTR) (11). The dysregulation of miRNAs is common in various carcinomas and plays an important role in cancer progression by altering normal gene expression $(12,13)$.

Previous studies have suggested that miR-155 is overexpressed in various solid tumors (14-16). High expression levels of miR-155 have been found to correlate with the poor prognosis of colorectal cancer and pancreatic tumors $(16,17)$. Moreover, miR-155 has been shown to enhance tumor invasion and migration, and acts as a mediator of EMT (18). All these lines of evidence are consistent with the notion that miR-155 functions as an oncogenic miRNA in human cancers.

However, the biological roles of miR-155 in CRC remain poorly understood. Therefore, in this study, the expression 
of miR-155 in CRC was examined by real-time PCR and the effects of miR-155 on cell proliferation, invasion and EMT were also investigated.

\section{Materials and methods}

Patients and tissue samples. Primary CRC tissue and matched normal adjacent colorectal mucosa were obtained from 76 patients who underwent colorectal cancer resection without pre-operative treatment at the First Department of General Surgery, The Affiliated Hospital of North Sichuan Medical College, Nanchong, China from 2009 to 2011, after receiving adequate informed consent. All tissue samples were immediately frozen in liquid nitrogen and stored at $-80^{\circ} \mathrm{C}$ until the extraction of RNA. The specimens were stained with hematoxylin and eosin and examined histopathologically. Sections that consisted of $>80 \%$ carcinoma cells were used to prepare the total RNA. Clinicopathological information, including age, gender, pathology, differentiation and tumor-node-metastasis (TNM) classification, was available for all patients. The study was approved by the Medical Ethics Committee of North Sichuan Medical College.

Cell culture. The SW480 human colon cancer cell line was obtained from the American Type Culture Collection (ATCC; Manassas, VA, USA) and cultured in RPMI-1640 medium (Gibco, Carlsbad, CA, USA) supplemented with heat-inactivated 10\% fetal bovine serum (FBS) (Invitrogen, Carlsbad, CA, USA), $100 \mathrm{U} / \mathrm{ml}$ penicillin and $100 \mathrm{mg} / \mathrm{ml}$ streptomycin, at $37^{\circ} \mathrm{C}$ in a humidified atmosphere of $5 \% \mathrm{CO}_{2}$ and $95 \%$ air.

Quantitative real-time reverse transcriptase-PCR ( $q R T-P C R)$. Total RNA was extracted from the tumor tissue and adjacent normal mucosa by homogenizing the tissue in TRIzol reagent (Invitrogen), according to the manufacturer's instructions. For miRNA qPCR, reverse transcription was performed using the QuantMir RT kit (System Biosciences, Mountain View, CA, USA). The cDNA then serves as the template for SYBR real-time PCR using Power SYBR-Green PCR Master mix (Applied Biosystems, Foster City, CA, USA). All reactions were run in triplicate on the iCycler iQ Multicolor Real-Time PCR Detection System (Bio-Rad, Hercules, CA, USA) using miR-155-specific primers (Applied Biosystems). The amplification profile was denatured at $95^{\circ} \mathrm{C}$ for $10 \mathrm{~min}$, followed by 40 cycles of denaturation at $95^{\circ} \mathrm{C}$ for $15 \mathrm{sec}$, annealing at $60^{\circ} \mathrm{C}$ for $30 \mathrm{sec}$, and extension at $72^{\circ} \mathrm{C}$ for $1 \mathrm{~min}$. The comparative cycle threshold $\left(\mathrm{C}_{\mathrm{T}}\right)$ method was applied to quantify the miRNA expression levels. The relative amount of miR-155 to small nuclear U6 RNA was calculated using the equation $2^{-\Delta C}{ }_{T}$ where $\Delta \mathrm{C}_{\mathrm{T}}=\left(\mathrm{C}_{\mathrm{T} \text { miR-155 }}-\mathrm{C}_{\mathrm{T} \text { U6 RNA }}\right)$. The fold change of gene expression was calculated using the $2^{-\Delta \Delta C T}$ method. U6 small nuclear RNA was used as the internal standard.

Transfection of miRNA. miR-155 precursor (pre-miR-155) and pre-miR miRNA precursor negative control (pre-miR-n.c.) were purchased from Applied Biosystems. For transfection, 2x10 cells were seeded into each well of a 6 -well plate. When the cells were $70-80 \%$ confluent the following day, they were transfected with pre-miR-155 and pre-miR-n.c. using Lipofectamine 2000 reagent (Invitrogen) following the manufacturer's instructions.
MTT assay. Following transfection of the SW480 cells with pre-miR-155 or pre-miR-n.c. for various periods of time (20 and $44 \mathrm{~h}$ ), the SW480 cells were re-seeded at a density of $2 \times 10^{4}$ cells/well in 96-well plates containing $0.2 \mathrm{ml} \mathrm{RPMI-1640}$ (with 10\% FBS). Subsequently, $20 \mu 1$ of 3-(4,5-dimethylthiazol2-yl)-2,5-diphenyltetrazolium bromide (MTT) were added followed by incubation for a further $4 \mathrm{~h}$ at $37^{\circ} \mathrm{C}$. A total of $150 \mu \mathrm{l}$ of dimethyl sulfoxide was then added to each well and the absorbance was measured at $570 \mathrm{~nm}$ on an enzyme immunoassay analyzer (Bio-Rad).

Migration and invasion assays. To measure cell migration and invasion, $5 \times 10^{4} \mathrm{SW} 480$ cells transfected with either pre-miR- 155 or pre-miR-n.c. were seeded into Transwell chambers $(8.0 \mu \mathrm{m}$ pore size; Corning Inc., Corning, NY, USA) uncoated or coated with Matrigel (BD Biosciences, Bedford, MA, USA).Medium containing $10 \%$ FBS in the lower chamber served as the chemoattractant. After the cells were incubated for $48 \mathrm{~h}$ at $37^{\circ} \mathrm{C}$ in a humidified incubator with $5 \% \mathrm{CO}_{2}$, cells that did not migrate through the pores were mechanically removed by a cotton swab. The migrated cells attached to the bottom of the membrane insert were fixed in methanol at room temperature for $5 \mathrm{~min}$ and stained with hematoxylin. The number of migrated or invaded cells on the lower surface of the membrane was then counted under a microscope at a magnification of $\mathrm{x} 400$.

Western blot analysis. To isolate the proteins, cells collected from 6-well plates were washed twice in PBS and lysed in RIPA lysis buffer (ProMab Biotechnologies, Inc., Richmond, CA, USA). The lysates were kept on ice for $30 \mathrm{~min}$, and then centrifuged at 13,000 $\mathrm{x}$ g for $30 \mathrm{~min}$. The surpernatant was collected and then $20 \mu \mathrm{g}$ of each of the proteins was separated by SDS-PAGE on $10 \%$ gels and transferred onto nitrocellulose membranes. After being blocked in 5\% skimmed milk, the membranes were incubated with the respective antibodies: goat anti-E-cadherin (Santa Cruz Biotechnology, Inc., Santa Cruz, CA, USA), goat anti-ZEB1 (Santa Cruz Biotechnology, Inc.), mouse anti-GAPDH (Santa Cruz, Biotechnology, Inc.) and rabbit anti-claudin-1 antibody (Zymed/Invitrogen). After incubation with the appropriate secondary antibody, the bands were visualized using ECL-Plus reagents (GE Healthcare Bio-Science Corp., Piscataway, NJ, USA). The density of the E-cadherin, ZEB1, claudin-1 and GAPDH bands was measured using Image J software, and values were normalized to the densitometric values of GAPDH in each sample. The fold change in protein amount was then calculated for the experimental sets compared to the control.

Statistical analysis. Continuous variables are expressed as the means $\pm \mathrm{SD}$. The gene expression levels in the CRC samples were compared with those in normal adjacent mucosa using the Wilcoxon test. Measurement data were analyzed using the Student's t-test, while categorical data were examined using the $\chi^{2}$ test. P-values $<0.05$ were considered to indicate statistically significant differences. All statistical analyses were performed using SPSS 16.0 software (SPSS; Chicago, IL, USA).

\section{Results}

Clinicopathological significance of miR-155 in CRC patients. Using qRT-PCR, we found that the miR-155 expression levels 
Table I. Association of miR-155 expression with the clinicopathological characteristics of colorectal cancer patients.

\begin{tabular}{|c|c|c|c|}
\hline \multirow[b]{2}{*}{ Characteristics } & \multicolumn{2}{|c|}{ miR-155 expression } & \multirow[b]{2}{*}{ P-value } \\
\hline & $\begin{array}{c}\text { Low } \\
(n=38)\end{array}$ & $\begin{array}{l}\text { High } \\
(n=38)\end{array}$ & \\
\hline Age (years) & $64.1 \pm 13.9$ & $62.3 \pm 11.3$ & 0.511 \\
\hline Gender & & & 0.222 \\
\hline Male & 28 & 23 & \\
\hline Female & 10 & 15 & \\
\hline Tumor size & & & 0.159 \\
\hline$\leq 5 \mathrm{~cm}$ & 26 & 20 & \\
\hline$>5 \mathrm{~cm}$ & 12 & 18 & \\
\hline Histological type & & & 0.243 \\
\hline Well, moderate & 25 & 20 & \\
\hline Poor, mucinous & 13 & 18 & \\
\hline Depth of invasion & & & 0.063 \\
\hline $\mathrm{T} 1, \mathrm{~T} 2$ & 20 & 12 & \\
\hline $\mathrm{T} 3, \mathrm{~T} 4$ & 18 & 26 & \\
\hline TNM stage & & & $0.020^{\mathrm{a}}$ \\
\hline I, II & 21 & 11 & \\
\hline III, IV & 17 & 27 & \\
\hline Location & & & 0.622 \\
\hline Colon & 11 & 13 & \\
\hline Rectum & 27 & 25 & \\
\hline Lymph node metastasis & & & $0.011^{\mathrm{a}}$ \\
\hline Absent & 23 & 12 & \\
\hline Present & 15 & 26 & \\
\hline Lymph node invasion & & & 0.169 \\
\hline Absent & 22 & 16 & \\
\hline Present & 16 & 22 & \\
\hline Distant metastasis & & & $0.033^{\mathrm{a}}$ \\
\hline Absent & 35 & 28 & \\
\hline Present & 3 & 10 & \\
\hline
\end{tabular}

${ }^{\mathrm{a}} \mathrm{P}<0.05$. n, number of patients.

were significantly upregulated in the cancer tissues from patients with CRC compared to those from the normal adjacent mucosa $(\mathrm{P}<0.01$, Fig. 1). The expression levels of miR-155 were categorized as low or high in relation to the median value. The high expression levels of $\mathrm{miR}-155$ in CRC with respect to several standard clinicopathological characteristics are presented in Table I. Our results demonstrated that the high expression of miR-155 significantly correlated with an advanced TNM stage, lymph node and distant metastasis $(\mathrm{P}<0.05$, Table I). However, there was no significant correlation between miR-155 expression and other clinicopathological characteristics, such as age, gender, tumor size, histological type, depth of invasion, tumor location, or lymph node invasion $(\mathrm{P}>0.05$, Table I).

miR-155 modulates migration and invasion but not proliferation in CRC cells. To investigate the mechanism by which miR-155 promotes lymph node and distant metastasis, we examined

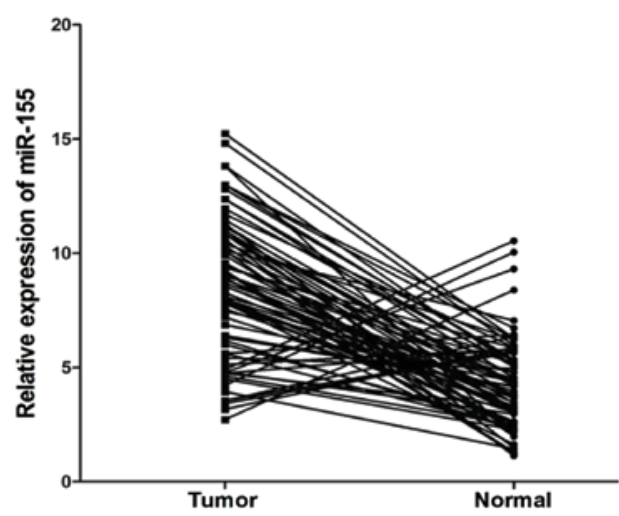

Figure 1. Comparison of microRNA-155 (miR-155) expression levels between tumor tissue and normal mucosa. The miR-155 expression levels were normalized using U6 as an internal control. The P-value was calculated using the Wilcoxon test. miR-155 expression levels were higher in the cancer tissue compared to the normal adjacent mucosa $(\mathrm{P}<0.01)$.

the role of miR-155 in CRC cell migration and invasion. Total RNA was isolated from the cells $48 \mathrm{~h}$ after transfection; the expression of miR-155 was significantly increased in the cells transfected with pre-miR-155, compared with that in the cells transfected with pre-miR-n.c. $(\mathrm{P}<0.01$, Fig. $2 \mathrm{~A})$. We observed that cell migration was significantly increased following transfection with pre-miR-155 compared with the negative control $(\mathrm{P}<0.01$, Fig. 2C). We then examined the effect of miR-155 on cell invasion across an extracellular matrix and showed that in the SW480 cells, the overexpression of miR-155 markedly enhanced the invasive potential compared with the control $(\mathrm{P}<0.01$, Fig. 2D). However, the overexpression of miR-155 in the SW480 cells had no significant effect on cell proliferation (P>0.05, Fig. 2B). These results demonstrate that miR-155 promotes CRC cell migration and invasion but has no effect on cell proliferation.

Upregulation of miR-155 alters cell morphology and inhibits E-cadherin and enhances ZEB1 expression in CRC cells. The SW480 cells transfected with pre-miR-155 underwent an EMT-like transformation, evidenced by the loss of cell-cell adhesion and an alteration of morphology, from a round compact shape to a spindle shape, which may facilitate cell migration (Fig. 3A). In addition, the expression of E-cadherin in the SW480 cells following transfection with pre-miR-155 was decreased, whereas the expression of ZEB1 was increased ( $P<0.01$, Fig. 3B and C). These effects of miR-155 on CRC cells may involve the induction of EMT. These findings demonstrate the potential role of miR-155 in promoting cell migration and invasion.

Regulation of claudin-1 expression by miR-155 in CRC cells. Through bioinformatics analyses with TargetScan version 6.2, we found that E-cadherin and ZEB1 are not the targets of miR-155. However, the 3'UTR of claudin-1 mRNA contains a highly conserved binding site of miR-155 (Fig. 4A), and claudin-1 is predicted to be a potential target for miR-155. To validate this hypothesis, we compared claudin- 1 expression in the SW480 cells transfected with pre-miR-155 or pre-miR-n.c. Indeed, the protein level of claudin-1 was increased in the SW480 cells transfected with pre-miR-155 compared with the control-transfected cells ( $\mathrm{P}<0.01$, Fig. 4B). 

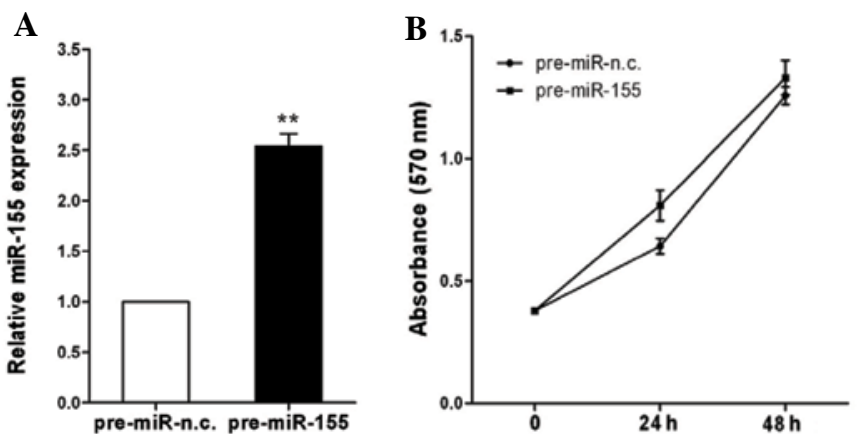

C
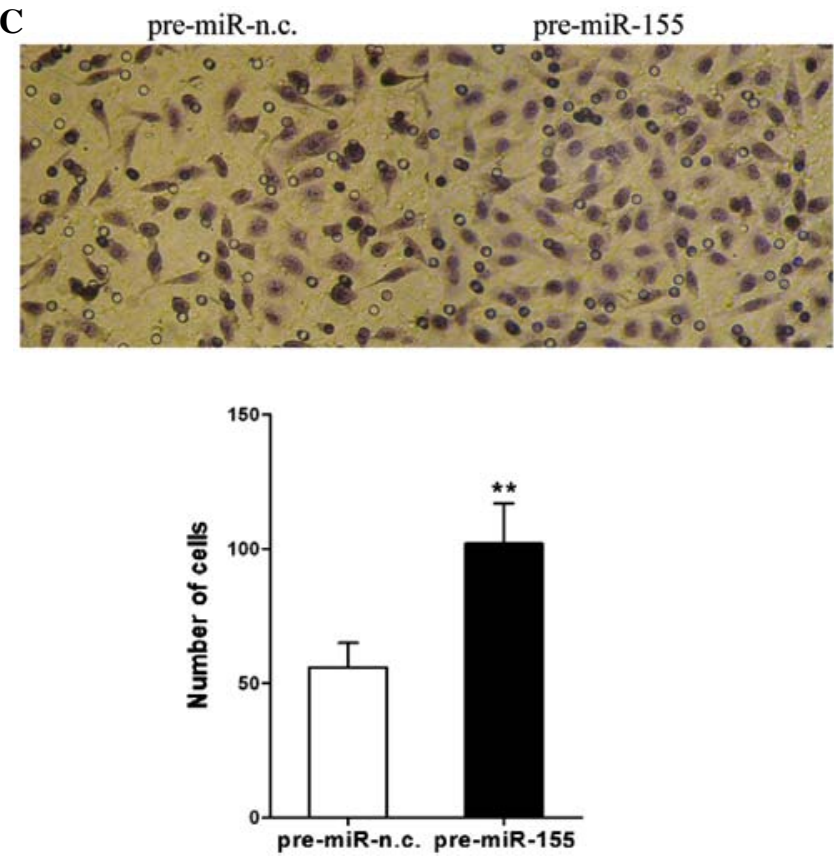

D
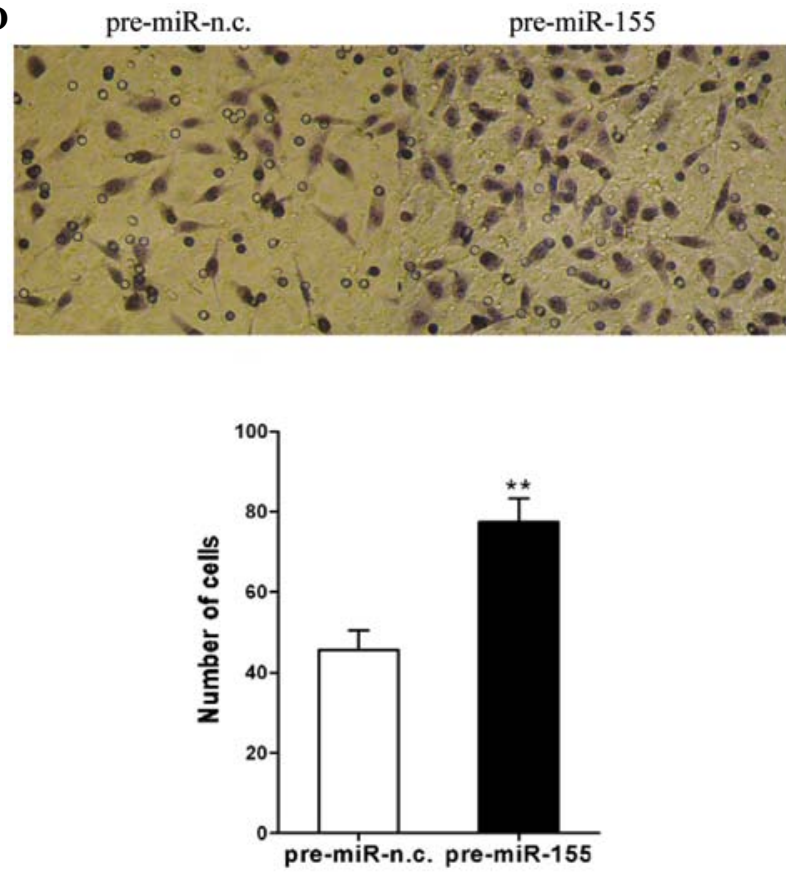

Figure 2. (A) MicroRNA-155 (miR-155) expression in SW480 cells transfected with pre-miR-155 or the negative control, pre-miR-n.c. miR-155 expression was determined by qRT-PCR. (B) MTT assay was performed to evaluate SW480 cell proliferation. (C) Effects of pre-miR-155 on the migration potential of SW480 cells. (D) Effects of pre-miR-155 on the invasive potential of SW480 cells. ${ }^{* *} \mathrm{P}<0.01$.
A pre-miR-n.c. pre-miR-155

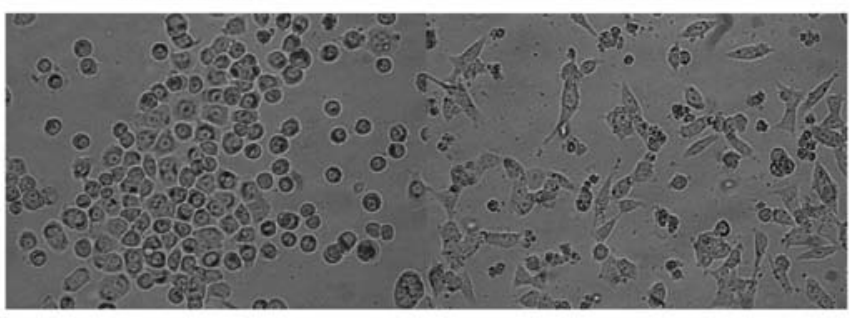

B
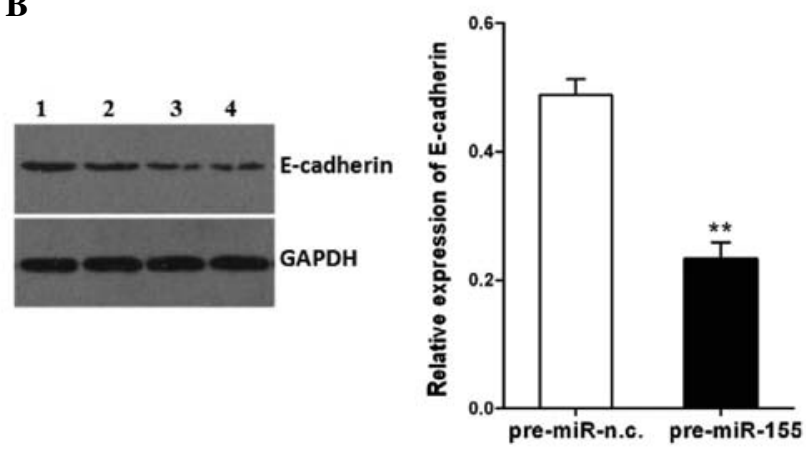

C
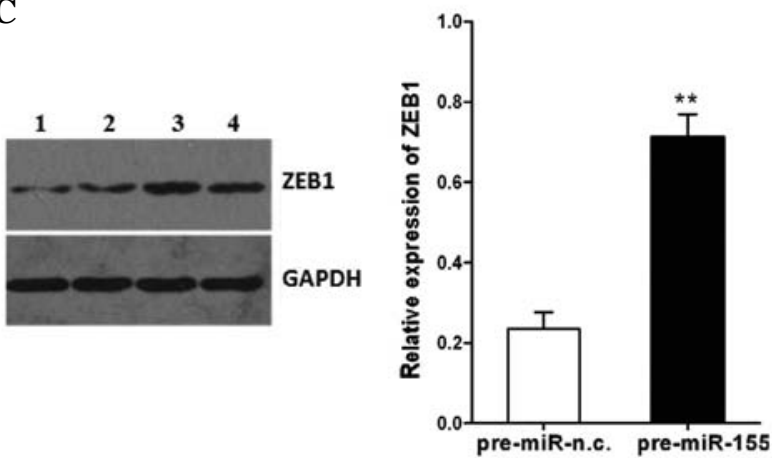

Figure 3. (A) The morphological changes of SW480 cells transfected with pre-miR-155 or the negative control, pre-miR-n.c. (B) E-cadherin protein expression was detected by western blot analysis. Lanes 1 and 2, SW480 cells transfected with pre-miR-n.c.; lanes 3 and 4, SW480 cells transfected with premiR-155. (C) ZEB1 protein expression was detected by western blot analysis. Lanes 1 and 2, SW480 cells transfected with pre-miR-n.c.; lanes 3 and 4 , SW480 cells transfected with pre-miR-155. ${ }^{* *} \mathrm{P}<0.01$.

A

Position 2528-2534 of claudin-1 3' UTR 5' ...UAGGAAUUUGUAUAAAGCAUUAC. hsa-miR-155 3' | || || ||

$\mathbf{B}$
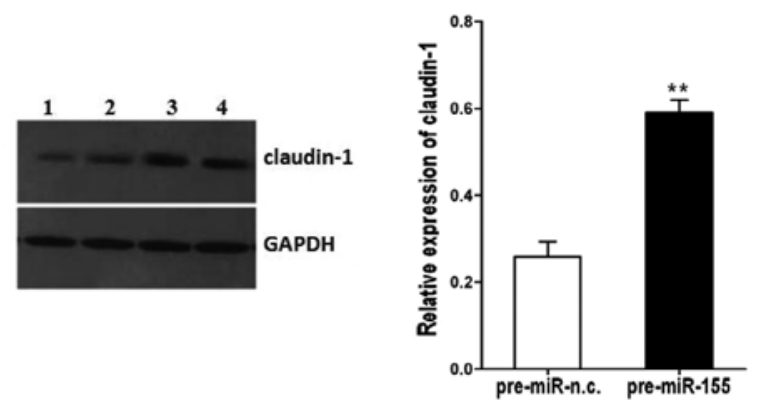

Figure 4. (A) MicroRNA-155 (miR-155) binding site in claudin-1 3'UTR, provided by TargetScan version 6.2. (B) Claudin-1 protein expression was detected by western blot analysis. Lanes 1 and 2, SW480 cells transfected with premiR-n.c.; lanes 3 and 4, SW480 cells transfected with pre-miR-155. ${ }^{* * *} \mathrm{P}<0.01$. 


\section{Discussion}

miR-155 is considered an important miRNA which is located in the BIC gene and is highly expressed in a variety of solid tumors, including breast, lung, colon, pancreatic and thyroid cancers (14-16). In the present study, we also confirmed that the expression of miR-155 was significantly increased in CRC tissues compared with normal adjacent mucosa, which suggests that the overexpression of miR-155 correlates with the carcinogenesis of CRC. Moreover, we found that miR-155 expression was associated with an advanced clinical stage and lymph node and distant metastases, strongly suggesting that miR-155 is involved in the development, progression and metastasis of colorectal cancer. However, Shibuya et al reported that the expression level of miR-155 only correlated with lymph node metastasis in colorectal cancer (17). These inconsistent results may be due to the use of different detection methods, specimen collection and patient clinicopathological data evaluation.

Cell motility and invasion are required for the dissemination of tumor cells from their primary location to lymph or blood vessels during metastasis (19). Our data demonstrate that miR-155 promotes cell migration and invasion, but not cell proliferation, which is consistent with our tissue correlation study in which the level of miR-155 was found to be associated with lymph node and distant metastasis, but not the $\mathrm{T}$ stage and tumor size. In addition, the results of this study revealed that tumors with a high expression of miR-155 had a spindle-like morphology, and that the upregulation of miR-155 decreased the expression of E-cadherin and increased the expression of ZEB1, suggesting that miR-155 is involved in the regulation of EMT. These findings demonstrate that miR-155 exerts its effects on CRC progression by promoting the metastatic ability of CRC cells.

The claudin family of proteins is the principal constituent of the tight junction (20). Claudins are dynamic proteins and help regulate a variety of cellular functions, such as proliferation, migration and EMT, in addition to their tight junction function (21-23). It has been reported that claudin-1 expression is highly upregulated and mislocalized in colon cancer and correlates with tumor progression and metastasis (24). Kinugasa et al also reported that claudin-1 levels were upregulated in hepatic metastatic lesions of colorectal cancer (25). In the present study, transfection with miR-155 significantly upregulated claudin-1 expression in CRC cells. Using TargetScan analysis, miR-155 was found to target the claudin-1 gene directly. Thus, the overexpression of miR-155 promotes the migration and invasive ability of CRC cells, at least in part through the regulation of claudin-1 expression. A previous study reported that claudin-1 upregulated the repressor ZEB-1, inhibiting E-cadherin expression in colon cancer cells (26). These data suggest that the ectopic expression of miR-155 upregulates claudin-1 expression, thus increasing ZEB-1 expression levels and inhibiting E-cadherin, thus facilitating cell migration and invasion. Thus, the data presented in this study suggest that miR-155 plays a role in the metastatic progression of CRC through the regulation of claudin-1 expression.

In conclusion, the overexpression of miR-155 plays an important role in the migration and invasion of CRC; its effects on CRC cells are possibly associated with the regulation of the claudin-1/ZEB-1/E-cadherin axis. The results from this study provide important clues as to the mechanisms involved in metastasis in CRC. However, further studies are required to fully elucidate the mechanisms involved in metastatic progression in CRC .

\section{Acknowledgements}

This study was supported by a grant from the Scientific Research Program of the North Sichuan Medical College of China (CBY11-A-ZP-05).

\section{References}

1. Jemal A, Siegel R, Ward E, et al: Cancer statistics. CA Cancer J Clin 58: 71-96, 2008.

2. Kobayashi H, Mochizuki H, Sugihara K, et al: Characteristics of recurrence and surveillance tools after curative resection for colorectal cancer: a multicenter study. Surgery 141: 67-75, 2007.

3. Yang $\mathbf{J}$ and Weinberg RA: Epithelial-mesenchymal transition: at the crossroads of development and tumour metastasis. Dev Cell 14: 818-829, 2008.

4. Jou J and Diehl AM: Epithelial-mesenchymal transitions and hepatocarcinogenesis. J Clin Invest 120: 1031-1034, 2010.

5. Spaderna S, Schmalhofer O, Hlubek F, et al: A transient, EMT-linked loss of basement membranes indicates metastasis and poor survival in colorectal cancer. Gastroenterology 131: 830-840, 2006

6. Thiery JP: Epithelial-mesenchymal transitions in development and pathologies. Curr Opin Cell Biol 15: 740-746, 2003.

7. Eades G, Yao Y, Yang M, et al: miR-200a regulates SIRT1 expression and epithelial to mesenchymal transition (EMT)-like transformation in mammary epithelial cells. J Biol Chem 286: 25992-26002, 2011.

8. Kurashige J, Kamohara H, Watanabe M, et al: MicroRNA-200b regulates cell proliferation, invasion, and migration by directly targeting ZEB2 in gastric carcinoma. Ann Surg Oncol 19 (Suppl 3): S656-S664, 2012.

9. Yang J, Mani SA, Donaher JL, et al: Twist, a master regulator of morphogenesis, plays an essential role in tumor metastasis. Cell 117: 927-939, 2004

10. Eger A, Aigner K, Sonderegger S, et al: DeltaEF1 is a transcriptional repressor of E-cadherin and regulates epithelial plasticity in breast cancer cells. Oncogene 24: 2375-2385, 2005.

11. Bartel DP: MicroRNAs: genomics, biogenesis, mechanism, and function. Cell 116: 281-297, 2004.

12. Zhang T, Liu M, Wang C, et al: Down-regulation of miR-206 promotes proliferation and invasion of laryngeal cancer by regulating VEGF expression. Anticancer Res 31: 3859-3863, 2011.

13. $\mathrm{Wu} \mathrm{J}, \mathrm{Wu} \mathrm{G}, \mathrm{Lv} \mathrm{L}$, et al: MicroRNA-34a inhibits migration and invasion of colon cancer cells via targeting to Fra-1. Carcinogenesis 33: 519-528, 2012.

14. Gironella M, Seux M, Xie MJ, et al: Tumor protein 53-induced nuclear protein 1 expression is repressed by miR-155 and its restoration inhibits pancreatic tumor development. Proc Natl Acad Sci USA 104: 16170-16175, 2007.

15. Iorio MV, Ferracin M, Liu CG, et al: MicroRNA gene expression deregulation in human breast cancer. Cancer Res 65: 7065-7070, 2005.

16. Yanaihara N, Caplen N, Bowman E, et al: Unique microRNA molecular profiles in lung cancer diagnosis and prognosis. Cancer Cell 9: 189-198, 2006.

17. Shibuya H, Iinuma H, Shimada R, et al: Clinicopathological and prognostic value of microRNA-21 and microRNA-155 in colorectal cancer. Oncology 79: 313-320, 2010.

18. Kong W, Yang H, He L, et al: MicroRNA-155 is regulated by the transforming growth factor beta/Smad pathway and contributes to epithelial cell plasticity by targeting RhoA. Mol Cell Biol 28: 6773-6784, 2008.

19. Bacac M and Stamenkovic I: Metastatic cancer cell. Annu Rev Pathol 3: 221-247, 2008

20. Tsukita $\mathrm{S}$ and Furuse M: Claudin-based barrier in simple and stratified cellular sheets. Curr Opin Cell Biol 14: 531-536, 2002. 
21. Ikari A, Takiguchi A,Atomi K, et al: Decrease in claudin-2 expression enhances cell migration in renal epithelial Madin-Darby canine kidney cells. J Cell Physiol 226: 1471-1478, 2011.

22. Yoon CH, Kim MJ, Park MJ, et al: Claudin-1 acts through c-Abl-protein kinase CI (PKCI) signaling and has a causal role in the acquisition of invasive capacity in human liver cells. J Biol Chem 285: 226-233, 2010.

23. Lee JW, Hsiao WT, Chen HY, et al: Up-regulated claudin-1 expression confers resistance to cell death of nasopharyngeal carcinoma cells. Int J Cancer 126: 1353-1366, 2010.
24. Dhawan P, Singh AB, Deane NG, et al: Claudin-1 regulates cellular transformation and metastatic or in colon cancer. J Clin Invest 115: 1765-1776, 2005.

25. Kinugasa T, Akagi Y, Ochi T, et al: Increased claudin-1 protein expression in hepatic metastatic lesions of colorectal cancer. Anticancer Res 32: 2309-2314, 2012.

26. Singh AB, Sharma A, Smith JJ, et al: Claudin-1 up-regulates the repressor ZEB-1 to inhibit E-cadherin expression in colon cancer cells. Gastroenterology 141: 2140-2153, 2011. 